\title{
Fatores determinantes para o acesso à informação por produtores de hortaliças na região sul de Moçambique
}

\author{
Determinants factors of access to information for vegetable \\ producers in southern Mozambique
}

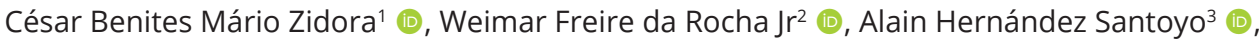
Miguel Angel Uribe-Opazo 4 (D)

\author{
1'Instituto Superior Politécnico de Gaza (ISPG), Divisão de Economia e Gestão, Chókwe (Gaza), Moçambique. \\ E-mail: cesarzidora@yahoo.com.br; cesarzidora@gmail.com \\ 2Programa de Pós-graduação em Desenvolvimento Regional e Agronegócio, Universidade Estadual do Oeste do Paraná \\ (UNIOESTE), Toledo (PR), Brasil. E-mail: wrochajr2000@gmail.com; wrochajr2000@yahoo.com.br \\ ${ }^{3}$ Programa de Pós-graduação em Economia, Universidade Federal de Alfenas (UNIFAL), Varginha (MG), Brasil. \\ E-mails: santoyocuba@gmail.com; alain.santoyo@unifal-mg.edu.br \\ ${ }^{4}$ Universidade Estadual do Oeste do Paraná (UNIOESTE), Cascavel (PR), Brasil. E-mail: miguel.opazo@unioeste.br
}

\begin{abstract}
Como citar: Zidora, C. B. M., Rocha Jr, W. F., Santoyo, A. H., \& Uribe-Opazo, M. A. (2022). Fatores determinantes para o acesso à informação por produtores de hortaliças na região sul de Moçambique. Revista de Economia e Sociologia Rural, 60(spe), e238628. https://doi.org/10.1590/1806-9479.2021.238628
\end{abstract}

Resumo: A informação na agricultura é relevante, pois mantém o produtor conectado a acontecimentos de natureza produtiva e comercial. O objetivo do artigo é identificar e analisar os fatores determinantes para o acesso à informação por produtores de hortaliças na região sul de Moçambique. A pesquisa foi explicativa, descritiva, com abordagem qualitativa, quantitativa, de natureza aplicada e de cunho primário e secundário. Para a coleta de dados em campo, aplicaram-se questionários a 384 produtores rurais que produzem e comercializam hortaliças, escolhidos aleatoriamente em uma amostragem probabilística estratificada. A pesquisa ocorreu entre fevereiro e junho de 2019, e a análise de dados foi descritiva e econométrica por meio do modelo logit binário. Como resultado, o perfil dos produtores foi de pessoas com idade entre 19 e 85 anos, dos quais 50\% não tinham formação escolar, formado por 46\% de homens e 54\% de mulheres que cultivavam em pequenas áreas de forma intensiva. As variáveis explicativas estatisticamente significativas e determinantes para o acesso à informação foram: idade; pertence a uma cooperativa/associação de produtores; experiência na produção; satisfação com o retorno financeiro; assistência técnica; acesso ao crédito; rendimento produzido por safra; uso de telefone na negociação; forma usada para comercializar a produção; e gestão da produção.

Palavras-chave: produtor familiar, comercialização agrícola, informação agrícola, logit binário.

\begin{abstract}
Information in agriculture is relevant, as it keeps the producer connected with events of a productive and commercial nature. The article aims to identify and analyze the determinants of access to information for vegetable producers in the southern region of Mozambique. The research was explanatory, descriptive, with a qualitative and quantitative approach and an applied nature. The research was primary and secondary. For data collection in the field, questionnaires were distributed to 384 rural producers who produce and commercialize vegetables. The participants were chosen randomly in stratified probabilistic sampling. The research took place between February and June 2019. Data analysis was descriptive and econometric, using the binary logit model. As a result, the profile of the producers was made up of people aged between 19 and 85 years, of which 50\% have no school education, composed of men (46\%) and women (54\%) who farm intensively in small areas. The statistically significant explanatory variables and determinants to access to information were: age, belonging to a cooperative/producer association, experience in production, satisfaction with financial return, technical assistance, access to credit, yield produced per harvest, use of the telephone in negotiation, the form used to commercialize the produce and production management.
\end{abstract}

Keywords: family producer, agricultural markets, agricultural information, binary logit. 


\section{INTRODUÇÃO}

Em Moçambique, as hortaliças são produzidas em todo o território nacional e durante todo o ano, tendo a região sul maior destaque (províncias de Maputo e Gaza) em razão das condições edafoclimáticas favoráveis e da experiência dos próprios produtores. As hortaliças proporcionam meios de subsistência que são resilientes a contrações econômicas e contribuem para o desenvolvimento econômico das cidades, da região e da comunidade produtora, proporcionando geração e distribuição da renda.

Para a Organização das Nações Unidas para Alimentação e Agricultura (2013), as hortaliças contribuem para a segurança alimentar e a melhoria da saúde humana, por serem as principais fontes de nutrientes, como vitaminas A e C, potássio, ácido fólico e fibra alimentar. A fibra dietética vegetal ajuda na redução dos níveis de colesterol e risco de doença cardíaca, essenciais para a digestão, além de ajudar a reduzir as doenças crônicas.

A renda de um horticultor, especificamente na região sul de Moçambique, está acima da linha nacional de pobreza, estimada em 1,90 dólar por dia (Organização das Nações Unidas para Alimentação e Agricultura, 2013; World Bank, 2018). Nesse sentido, a produção nacional de hortaliças atinge cifras maiores em termos de produtividade quando comparada com as demais culturas. No aspecto econômico, a produção de hortaliças propicia ao produtor familiar uma renda, contribuindo para o bem-estar social.

A produção é dominada por pequenos agricultores, que chegam a atingir níveis de produtividade relativamente altos, muitos deles próximos aos padrões internacionais, como o caso do tomate, segundo o Ministério de Agricultura e Segurança Alimentar (MASA) (Moçambique, 2017), e, consequentemente, beneficiam-se das receitas. No processo de comercialização das hortaliças, é possível agregar valor ao produto e, como consequência, proporcionar renda à população rural, tendo um papel relevante para a economia local e nacional, uma vez que um grande contingente de pessoas tem sua vida ligada à agricultura em Moçambique.

O produtor moçambicano, para gerenciar a produção de forma eficaz, rentável e competitiva, precisa de informações relacionadas ao plantio - expectativa de preços, preços de produtos competitivos, produtividade esperada, área disponível e adoções de pacotes tecnológicos para responder às questões: o que, quanto, quando, onde e como produzir, de que forma distribuir os produtos e ainda conhecer os preços de insumos e de venda. Possuir informação constitui um elemento decisivo, visto que o processo de produção está conectado a algum grau de conhecimento e ao processamento de informação. A geração da informação do processo produtivo e a sua perfeita circulação entre os demais agentes no ramo agrícola determinam o grau de sucesso na atividade.

As informações agrícolas oferecem oportunidades para os formuladores de políticas avaliarem o desempenho dos mercados de produtos agrícolas e determinarem as restrições microeconômicas. Por outro lado, a informação sobre o mercado é particularmente importante na provisão de alertas sobre a escassez de alimentos e ainda no gerenciamento de reservas de segurança alimentar (Alemu et al., 2006). Ademais, de acordo com o MASA (Moçambique, 2017), para a viabilização da produção de hortaliças no setor familiar, é necessário dotar os produtores de gestão de produção e conhecimento de mercados ao seu alcance para fortalecer e estreitar a oferta e a demanda.

Para Schneider (2016), o mercado, no sentido amplo, pode ser entendido como uma construção social, um espaço de interação e troca, regido por normas e regras de caráter formal ou informal, em que são emitidos sinais como os preços, que detêm ou influenciam as decisões dos atores envolvidos. O autor faz simultaneamente uma classificação dos mercados em quatro tipos genéricos: mercado de proximidade; mercados locais e territoriais; mercados convencionais; 
e, por fim, mercados públicos e institucionais. Dos mercados listados, pelas características, o mais usado pelo produtor do setor familiar em Moçambique é o de proximidade.

A maior dificuldade enfrentada pelos produtores rurais, principalmente pelos pequenos e médios, no processo de produção e comercialização agrícola é o fato de terem poucas informações sobre a atividade em si, visto que muitos deles dispõem de menor número de informações, ficando, assim, dependentes dos agentes de mercado para comercializar sua produção (Schneider, 1990). A constatação feita por Schneider é extensiva aos produtores de hortaliças em Moçambique, já que existem problemas relacionados à fraca circulação ou mesmo à ausência de informação agrícola em mercados, quer de insumos ou mesmo de consumidores, afetando a viabilidade tanto da produção como da comercialização.

Para Machado (2014), o setor das hortaliças desempenha um papel crucial na vida do produtor em Moçambique. Seria necessário haver, então, ações do governo a curto/médio prazo no que diz respeito ao uso de políticas dinâmicas, com vistas a melhorar o sistema de informação para assegurar a disponibilidade dos produtos no momento certo, no lugar certo e com o preço certo, de modo a satisfazer os consumidores cada vez mais exigentes.

O objetivo deste artigo é identificar e analisar os fatores determinantes do acesso à informação dos produtores de hortaliças na região sul de Moçambique. As hortaliças nesse país têm relevância econômica, sendo produzidas em volume significativo e com um grande contingente de pessoas envolvidas na produção.

Para atingir os objetivos da pesquisa, foram aplicados 384 questionários aos produtores de hortaliças da região sul de Moçambique, no período compreendido entre 20 de fevereiro e 30 de junho de 2019. A análise dos dados foi feita por meio do modelo econométrico logit binário. Usou-se o modelo para testar hipóteses a respeito de fatores determinantes do acesso à informação na produção e comercialização de hortaliças. A variável dependente foi o acesso à informação, qualitativa e discreta, que assumiu os valores binários 0 (se não tiver acesso à informação) ou 1 (se tiver acesso à informação).

Para o desenvolvimento deste estudo, fez-se uma divisão em cinco seções. A primeira constitui a presente introdução, que apresenta a visão panorâmica do universo estudado; já na segunda seção, há o referencial teórico, que deu suporte a esta pesquisa; na sequência, discute-se a metodologia aplicada, na qual serão detalhados os passos e as etapas da construção da pesquisa; a quarta seção apresenta os resultados e discussões desta pesquisa; e, por fim, a última seção é o fechamento deste texto por meio da conclusão.

\section{Revisão da literatura}

\subsection{Importância da informação e assimetria informacional}

A informação recebe uma gama de aceitação como um recurso essencial para a comunicação e a construção de conhecimentos. O uso de informações apropriadas garante o funcionamento individual e organizacional. A principal função de manter-se informado é basicamente aumentar o conhecimento e reduzir a incerteza (Adio et al., 2016). O'Donnell (2009) argumenta que, com a informação certa, é possível saber o que o cliente espera, o que a sua concorrência está oferecendo e o que se pode prometer entregar, além de quando e a que preço.

O destino de qualquer agregado de informação, segundo Barreto (1996), expressa-se em três equações básicas: a) $K=f(I)$, o conhecimento é uma função da informação; $b) D=f(K)$, o desenvolvimento do indivíduo e da sociedade é função do conhecimento acumulado como 
estabelecido por elementos da teoria econômica e da própria condição humana; e, assim, operando $a$ e $b, c) D=f(I)$, o desenvolvimento é uma função da informação.

Quando se priva o acesso à informação de um bem ou serviço, surge a assimetria de informação como uma consequência do mau fluxo de informação entre os agentes envolvidos em uma transação, sendo tipicamente um deles o comprador possuir informações menos perfeitas do que os outros (Williamson, 1985; Stiglitz, 2001). Segundo Rocha Junior (2004) e Rocha Junior \& Ribeiro (2011), a eficiência informacional resultante de uma distribuição simétrica da informação entre os agentes do mercado faz com que o mecanismo do mercado agregue simetricamente as informações disponíveis na economia, mitigando incertezas e promovendo um ambiente confiável, reduzindo os custos de transações.

Stiglitz (2001) aponta dois tipos de assimetria de informação: risco moral, que surge quando a parte detentora de mais informação, o vendedor, tira vantagem da assimetria de informação e pede um preço superior ao do equilíbrio do mercado; e seleção adversa, que se reflete no fato de um bem de qualidade inferior atingir preço superior que o de alta qualidade.

Segundo Williamson (1985), como consequência da assimetria de informação, existem dois pressupostos comportamentais entre os agentes envolvidos em uma transação: racionalidade limitada, que é a incapacidade que os agentes têm de prever e processar todas as contingências futuras em uma negociação; e oportunismo, que seria o comportamento do agente em querer tirar vantagens próprias na negociação por deter informações privilegiadas que outro não tem.

\subsection{Relevância da informação na agricultura}

Em mercados agrícolas, as informações são aquelas que podem ajudar os tomadores de decisões na produção e comercialização de produtos, sendo que a necessidade do consumidor é o elemento que induz todo o processo de produção. Em simultâneo, a informação é cada vez mais um fator crítico de sucesso em empreendimentos agrícolas, com especial atenção ao produtor familiar. Os agricultores, para poderem maximizar os seus objetivos, precisam ter conhecimento oportuno das decisões que vão sendo tomadas e capacidade para interpretá-las e agir em conformidade, em decisões de curto, médio e longo prazo (Batalha, 2014; Instituto Interamericano de Cooperação para a Agricultura, 2016).

A informação, segundo Lachica et al. (2008), precisa ser: a) confiável e imparcial, refletindo objetivamente as condições do mercado; b) oportuna, estando disponível aos usuários no menor tempo possível depois da sua coleta; c) relevante, sendo representativa para o produtor e o comprador, baseada na importância do produto e no que é estratégico para o mercado; d) acessível, estando disponível a todos os interessados e assegurando que sua utilização não crie privilégios.

No ramo agrícola, em especial em mercados de insumos e consumidor, as informações visam, sobretudo, à transparência, ou seja, à quantidade de informações de que se possa dispor sobre um produto específico. Quanto maior a disponibilidade de informações sobre um produto, maior será a transparência a seu respeito (Instituto Interamericano de Cooperação para a Agricultura, 2016). Para Stiglitz (2001), os benefícios da informação no mercado aumentam com a escala de sua produção (utilização).

O setor familiar tem desempenhado um papel crucial na produção e disponibilização de alimentos em quantidades e qualidades diante do crescimento populacional, contribuindo, dessa forma, para a segurança alimentar e nutricional da população mundial. Destaca-se também na luta global contra a fome que atinge mais de 800 milhões de pessoas. Em relação a isso, a tomada de decisão do produtor rural deixou de ser baseada na tradição, experiência 
e intuição, passando a ser apoiada por informações precisas em tempo real e pelo uso de tecnologias da informação (Silva, 2019).

\subsection{Caracterização da produção e comercialização de hortaliças em Moçambique}

Segundo Haber et al. (2015), a cadeia agroalimentar de hortaliças em Moçambique desempenha um considerável papel na geração e na distribuição da renda à agricultura familiar. Quanto à produção, os melhores índices foram atingidos entre o período de 2007 a 2013. No ano de 2007, foram produzidas 500 mil toneladas de hortaliças; em 2010, a produção chegou a atingir cerca de 900 mil toneladas, com destaque para as principais hortaliças, como tomate, cebola, pimentão, pepino, cenoura, quiabo, alho, alface, vagem verde e repolho, que contribuíram para tais índices de produção.

A produção é feita pelo produtor familiar e tem espaço em todo o território moçambicano, mas a região que se destaca é a sul do país (províncias de Maputo e Gaza), que tem contribuído com uma fração maior quando comparada com as demais regiões. A região sul funciona como o centro na produção e no fornecimento das hortaliças para o mercado nacional.

A rede de comercialização moçambicana para hortaliças é constituída por comerciantes informais (mercados municipais, mercados suburbanos, barracas e vendedores ambulantes) e comerciantes formais (mercearias e supermercados), que têm ganhado espaço, principalmente, nas cidades emergentes. A maior parte das transações de produtos agrícolas baseia-se nas relações entre pessoas, sendo que continuam ocorrendo por meio de trocas diretas em mercados físicos, e as economias de escala na comercialização não são totalmente exploradas (Haber et al., 2015).

O mercado interno de comercialização das hortaliças é caracterizado por possuir um baixo poder de compra em geral e uma grande incidência de pobreza, desenvolvendo a atividade de uma forma quase integralmente "informal". O mercado abastecedor de hortaliças em Moçambique encontra-se praticamente confinado aos grandes regadios na zona sul (Haber et al., 2015).

\section{Metodologia}

A pesquisa foi de natureza aplicada. Quanto ao objetivo, é descritiva e explicativa, e sua abordagem é qualitativa e quantitativa. Ela esteve concentrada na região sul de Moçambique: na província de Maputo, nos distritos de Moamba, Boane, Namaacha, Marracuene e Manhiça; e na província de Gaza, nos distritos de Bilene, Chókwè, Xai-Xai e Chibuto. O grupo-alvo do estudo foi toda a comunidade de produtores que produzem e comercializam hortaliças em uma amostra de 384 produtores, extraída de uma população total de 166.867 produtores. A escolha deles foi feita de forma aleatória nas suas unidades de produção. A pesquisa foi realizada entre 20 de fevereiro e 30 de junho de 2019. As hortaliças produzidas foram: tomate, pimentão, cebola, cenoura, repolho, feijão-verde, feijão-manteiga, alho, pepino, abóbora, couve, batata-inglesa e alface.

Para Haber et al. (2015), a maior parte das transações de produtos agrícolas em Moçambique baseia-se nas relações entre pessoas e de maneira informal. Segundo a constatação dos autores, em função das características da comercialização das hortaliças com o produtor familiar, podese dizer que está presente um mercado de proximidade, o qual, segundo Schneider (2016), tem como canais de comercialização: propriedade (colhe-pague), domicílio/casa, beira de estrada, entrega direta, feira local e grupos de consumo. Os canais propostos por Schneider (2016) se assemelham aos usados pelo produtor familiar de Moçambique ao comercializar a produção. 
A pesquisa foi primária (coleta de dados em campo) e secundária (consulta bibliográfica). A técnica de coleta de dados primários foi a aplicação de questionário aos produtores, o qual apresentava perguntas fechadas, escolha múltipla e algumas de escala de likert. Os produtores que se expressavam fluentemente em língua portuguesa preenchiam os questionários; caso contrário, as questões eram formuladas oralmente pelo pesquisador, e o produtor as respondia. O questionário estava dividido em três partes: I) informações sobre o entrevistado e a propriedade; II) informações sobre a produção e a comercialização agrícola; III) informações sobre mercados agrícolas. Para as análises dos dados, usou-se o pacote estatístico Eviews no nível de significância de $5 \%$ de probabilidade.

A população da pesquisa foi maior que 100 mil; assim, usou-se a seguinte fórmula matemática para definir a amostra, proposta por Israel (1992): $n=\frac{z^{2}}{e^{2}} p q$ (1). Adotou-se o $p=0,5$ (variabilidade máxima), com nível de confiança de $95 \%$, erro $\pm 0,05$ e $Z_{c}=1,96$. O tamanho da amostra a partir da equação foi: $\boldsymbol{n}=\frac{1.96^{2}}{0.05^{2}} 0.5 *(1-0.5)=384$. O método de amostragem para a seleção da amostra por distrito foi probabilístico estratificado (Tabela 1).

Tabela 1. Tamanho da amostra por distrito

\begin{tabular}{ccc} 
Distritos & Número de produtores & Tamanho da subamostra \\
Boane & 22314 & 51 \\
Moamba & 10000 & 23 \\
Namaacha & 10000 & 23 \\
Marracuene & 12127 & 28 \\
Manhiça & 30000 & 69 \\
Xai-Xai & 30000 & 69 \\
Bilene & 10000 & 23 \\
Chókwè & 12000 & 28 \\
Chibuto & 30426 & 70 \\
Total & $\mathbf{1 6 6 8 6 7}$ & $\mathbf{3 8 4}$ \\
\hline
\end{tabular}

Para inferir os resultados da pesquisa em função da amostra observada, usou-se o modelo logit binário. Segundo Gujarati \& Porter (2011), um dos principais objetivos no uso desse modelo é calcular a probabilidade de um indivíduo, com determinado conjunto de atributos, tomar uma decisão sobre a ocorrência de um dado evento.

A fim de analisar o acesso à informação ao produtor e dos possíveis determinantes na pesquisa, determinou-se a probabilidade do acesso à informação. A variável dependente é o acesso à informação, binária ou categórica igual a um $(Y=1)$, se os produtores tivessem acesso à informação, e zero, caso contrário $(Y=0)$. $O$ acesso à informação em campo foi determinado mediante a percepção do entrevistador para com os entrevistados, pois havia perguntas no questionário de raciocínio lógico feitas aos produtores que facultavam a conclusão se eles tinham ou não acesso à informação.

Até então, nas condições e realidade moçambicana, nenhum estudo semelhante foi realizado para análises dessas variáveis no setor das hortaliças. Assim, a escolha das variáveis independentes e, por conseguinte, os possíveis fatores determinantes para o acesso à informação basearamse em estudos empíricos realizados por pesquisadores de outros países (Quadro 1). O modelo econométrico usado nessas pesquisas foi o logit binário, à semelhança da presente pesquisa. 
Quadro 1. Estudos empíricos e sinal esperado

\begin{tabular}{|c|c|c|}
\hline Variável & Estudos empíricos que utilizaram a variável & $\begin{array}{l}\text { Sinal } \\
\text { esperado }\end{array}$ \\
\hline Idade do produtor (IP) & $\begin{array}{l}\text { Santos Filho (2008); Couto \& Alves (2013); Carrer et al. } \\
\text { (2013); Nchembi (2017); Dhraiefa et al. (2018); Melesse } \\
\text { (2018). }\end{array}$ & - \\
\hline Sexo do produtor (SP) & Couto \& Alves (2013); Lemessa et al. (2019). & + \\
\hline $\begin{array}{l}\text { Pertence à cooperativa/ } \\
\text { associação (PC) }\end{array}$ & $\begin{array}{l}\text { Lanna et al. (2011); Carrer et al. (2013); Aikin (2014); } \\
\text { Rajendran et al. (2016); Nchembi (2017); Theophilus et al. } \\
\text { (2019). }\end{array}$ & + \\
\hline $\begin{array}{l}\text { Tamanho do agregado } \\
\text { familiar (TAF) }\end{array}$ & Paulo (2011); Melesse (2018); Lemessa et al. (2019). & + \\
\hline $\begin{array}{l}\text { Tamanho da área } \\
\text { produzida (TAP) }\end{array}$ & Dias (1997); Ghimire et al. (2015); Melesse (2018). & + \\
\hline $\begin{array}{l}\text { Experiência na produção } \\
\text { de hortaliças (EPH) }\end{array}$ & $\begin{array}{l}\text { Dias (1997); Nchembi (2017); Dhraiefa et al. (2018); } \\
\text { Theophilus et al. (2019). }\end{array}$ & + \\
\hline $\begin{array}{l}\text { Distância do local da } \\
\text { produção (DLPM) }\end{array}$ & Rajendran et al. (2016); Lemessa et al. (2019). & - \\
\hline $\begin{array}{l}\text { Participação do dia de } \\
\text { campo (PDC) }\end{array}$ & Ghimire et al. (2015). & + \\
\hline $\begin{array}{l}\text { Satisfação com o retorno } \\
\text { financeiro (SRF) }\end{array}$ & Padilha Junior (2006). & + \\
\hline Assistência técnica (AT) & $\begin{array}{l}\text { Oliveira et al. (2005); Lanna et al. (2011); Melesse (2018); } \\
\text { Ghimire et al. (2015); Rajendran et al. (2016); Nchembi } \\
\text { (2017); Dhraiefa et al. (2018); Theophilus et al. (2019). }\end{array}$ & + \\
\hline Acesso ao crédito (AC) & Oliveira et al. (2005); Melesse (2018). & + \\
\hline $\begin{array}{l}\text { Rendimento da produção } \\
\text { (RP) }\end{array}$ & $\begin{array}{l}\text { Ghimire et al. (2015); Rajendran et al. (2016); Nchembi } \\
\text { (2017). }\end{array}$ & + \\
\hline $\begin{array}{l}\text { Renda bruta da } \\
\text { produção (RBP) }\end{array}$ & Bati (2017); As-Sunny et al. (2018). & + \\
\hline $\begin{array}{l}\text { Uso de pacote } \\
\text { tecnológico (UPT) }\end{array}$ & Carrer et al. (2013); Ghimire et al. (2015). & + \\
\hline $\begin{array}{l}\text { Uso de telefone na } \\
\text { negociação (UTN) }\end{array}$ & Dias (1997); Aikin (2014). & + \\
\hline $\begin{array}{l}\text { Gestão da propriedade } \\
\text { (GP) }\end{array}$ & Abas (2016). & + \\
\hline $\begin{array}{l}\text { Nível escolar do produtor } \\
\text { (NEP) }\end{array}$ & Oliveira et al. (2005); Lanna et al. (2011). & + \\
\hline $\begin{array}{l}\text { Forma usada para a } \\
\text { comercialização (FUC) }\end{array}$ & Callado (2011); Abas (2016). & + \\
\hline
\end{tabular}

Fonte: elaborado pelos autores.

\subsection{Descrição das variáveis}

Idade do produtor (IP): variável quantitativa medida em anos do produtor. Carrer et al. (2013) usaram a mesma variável como fator determinante em sua pesquisa. A variável mostrou sinal negativo, significando que, com o aumento da idade, havia a diminuição da probabilidade de o produtor demandar crédito por pecuaristas de corte no estado de São Paulo, isto é, os agricultores mais jovens tinham maior chance de acesso ao crédito que os mais velhos. 
Sexo do produtor (SP): variável dummy0, se for mulher, e 1, se for homem. Os agregados familiares chefiados por homens têm melhores oportunidades de acesso a ferramentas inerentes à produção do que as mulheres, uma vez que estão em constante exposição a novas informações e tendem a assumir riscos. Couto \& Alves (2013) usaram a mesma variável, tendo encontrado um sinal positivo, inferindo que as famílias chefiadas por mulheres eram mais propensas à pobreza em comparação às chefiadas por homens.

Pertence a uma cooperativa/associação (PC/A): variável qualitativa dummy 1 , se fizer parte de uma cooperativa, e 0 , caso contrário. A filiação do produtor em associação/cooperativa constitui uma ação coletiva benéfica, fato que ajuda o produtor a alcançar objetivos que, individualmente, seriam difíceis. Carrer et al. (2013) usaram a mesma variável em sua pesquisa, tendo constatado uma relação positiva, significando que produtores organizados em cooperativas/associações tinham mais chances sobre a demanda do crédito.

Tamanho do agregado familiar (TAF): variável quantitativa discreta, medida por número do agregado familiar. Entende-se que, quanto maior for o tamanho do agregado familiar, maior serão as despesas e os custos para garantir uma vida digna à família. Assim, com o aumento de agregado familiar, o produtor busca estratégias que visam garantir a rentabilidade da atividade e a geração de renda. Lemessa et al. (2019), em sua pesquisa, obteve uma relação positiva, indicando que famílias numerosas tinham maiores probabilidades na adoção de tecnologias altamente produtivas na cultura de batata.

Tamanho da área produzida (TAP):variável quantitativa medida por número de hectares. A produção por unidade de área passou a ser um critério mais importante para o rendimento potencial das culturas. Esta variável foi usada por Ghimire et al. (2015), e o resultado da análise foi um sinal positivo, indicando que, quanto mais extensa fosse a área produzida, maior seria a probabilidade na escolha de variedades de arroz altamente produtivas.

Experiência na produção de hortaliças (EPH): variável quantitativa medida por anos de produção. A experiência na produção é fator crucial e, por vezes, de sucesso, isto é, quanto mais tempo/experiência tiver o produtor na produção, maiores serão os ganhos produtivos. Paustian \& Theuvsen (2016), em sua pesquisa, usaram a mesma variável e encontraram sinal positivo, ou seja, quanto mais experiente fosse o produtor, maior seria sua probabilidade de adotar tecnologias altamente produtivas, garantindo, dessa forma, ganhos substanciais na geração de renda.

Distância entre o local de produção e o mercado (DLPM):variável quantitativa medida em quilômetro $(\mathrm{km})$. Usando os princípios da nova geografia econômica, a localização das unidades de produção e centros de consumo deve ser estratégica, isto é, a distância menor possível, pois isso ajuda a minimizar os custos de transporte da produção. Rajendran et al. (2016), em sua pesquisa, usaram essa variável, tendo constatado uma relação negativa que indica que unidades de produção localizadas longe do centro de consumo tinham menor probabilidade de adotar tecnologia agrícola sustentável.

Participação em dias de campo (PDC): variável qualitativa dummy 1, se participar, e 0, caso contrário. Segundo MINAG (Ministério de Agricultura, 2011), participar de atividades em dias de campo é benéfico para o produtor. Os dias de campo servem de ponte para a introdução e a transferência de uma nova tecnologia produtiva. Ghimire et al. (2015) usaram a mesma variável em sua pesquisa, tendo constatado um sinal positivo na análise, evidenciando que todos os produtores participantes nesse dia tinham maior probabilidade de acesso à informação e novas tecnologias a respeito da produção.

Satisfação com o retorno financeiro (SRF):variável qualitativa dummy 1 , se estiver satisfeito, e 0 , caso contrário. O retorno financeiro constitui algo motivacional para o produtor, visto 
que representa ganho econômico e boa motivação no exercício da atividade. Padilha Junior (2006) afirma que o retorno financeiro é crucial, visto que agrega valor e ainda torna o sistema de comercialização eficiente. Nesta variável, espera-se uma relação positiva, pois, quanto mais satisfeito com o retorno financeiro o produtor estiver, maior será a motivação e, em simultâneo, a probabilidade de ter acesso à informação.

Assistência técnica (AT): variável qualitativa dummy 1 , se tiver assistência, e 0, caso contrário. Acredita-se que os agricultores que recebem visitas dos agentes de extensão sejam expostos a novas tecnologias e informações atualizadas da produção e comercialização. Lanna et al. (2011) usaram a mesma variável em seu estudo Determinantes da adoção da tecnologia de despolpamento na cafeicultura: estudo de uma região produtora da Zona da Mata de Minas Gerais, e constataram um sinal positivo, evidenciando que os produtores com assistência técnica estavam mais propensos a adotar novas técnicas produtivas na cultura de café.

Acesso ao crédito $(A C)$ : variável qualitativa dummy 1, se tiver acesso, e 0 , caso contrário. $\mathrm{O}$ acesso ao crédito reduz os problemas de liquidez que o produtor enfrenta quando pretende comprar insumos agrícolas e, portanto, abre caminho para a aplicação oportuna de insumos, aumentando, assim, a produtividade geral e a renda. Espera-se um sinal positivo. Estudo feito por Oliveira et al. (2005) usou essa variável e obteve sinal positivo, concluindo que o crédito rural é um fator conjuntural que exerce o papel de promotor da modernização da agricultura.

Rendimento da produção $(R P)$ : variável quantitativa medida em quilograma por hectare (kg/ha). O exercício econômico de toda a produção agrícola é em função do rendimento obtido/ha e do preço de venda. Rendimentos baixos inesperados são inviáveis. Ghimire et al. (2015) usaram essa variável em sua pesquisa e obtiveram um sinal positivo, evidenciando que um maior rendimento em campo proporcionou maiores chances de os produtores adotarem técnicas produtivas sustentáveis na cultura de arroz em Nepal.

Renda bruta na produção de hortaliças (RBP): variável quantitativa medida em valor monetário (reais). A renda bruta obtida no exercício de uma atividade agrícola determina o grau do sucesso e insucesso em geral. Bati (2017) e As-Sunny et al. (2018) usaram a mesma variável e obtiveram sinal positivo, tendo, em seguida, concluído que, quanto maior fosse a renda obtida, maior seria a chance de os produtores adotarem técnicas altamente produtivas para rentabilizar a atividade.

Uso de pacote tecnológico de produção (APT): variável qualitativa dummy 1 , se fizer uso, e 0 , caso contrário. O princípio da adoção de tecnologia na produção agrícola é para aumentar os níveis de produção em quantidade e qualidade e, de certo modo, exige do produtor algum conhecimento e informação sobre tecnologia. Ghimire et al. (2015) usaram a mesma variável em seu estudo e obtiveram um sinal positivo, evidenciando que o uso da tecnologia na produção, por parte dos produtores, aumentava a probabilidade de eficiência na produção, visando a maiores ganhos.

Uso de telefone na negociação (UTN): variável qualitativa dummy 1 , se fizer uso, e 0 , caso contrário. $\mathrm{O}$ uso de telefone agrega valor no processo de produção das hortaliças, visto que em menos tempo e espaço o produtor consegue obter informação necessária sem maior esforço e gasto. Dias (1997) usou essa variável no estudo sobre o papel da informação de mercado na comercialização de hortigranjeiros no Distrito Federal, obteve um sinal positivo e constatou um aumento de probabilidade no acesso à informação para aqueles produtores que usavam ou tinham acesso ao telefone. 
Gestão da produção (GP): variável nominal medida em escala de likert. A gestão por si só constitui uma tarefa que demanda do gestor boa habilidade e competência. A aquisição da competência e da habilidade requer a posse de conhecimento e informação. Abas (2016), em sua pesquisa Factors Influencing Self-Reliance in Rice Production, The Case of Small Farmers in Bataan, Philippines, obteve uma relação positiva, indicando que boa gestão da produção contribuía para a autossuficiência na cultura de arroz.

Nível escolar do produtor (NEP): variável qualitativa ordinal medida por nível acadêmico concluído. Acredita-se que os agricultores instruídos tenham mais chances de realizar uma atividade de produção racional e eficiente. Oliveira et al. (2005) e Lanna et al. (2011) usaram essa variável em suas pesquisas e obtiveram um sinal positivo, evidenciando que, quanto mais instruído fosse o produtor, maior seria a probabilidade de adoção de novas tecnologias produtivas.

Forma usada para a comercialização (FUC): variável nominal. Quando a venda é feita em mercados informais, não há tanta exigência na busca de informação e conhecimento nem no uso de contratos. Mas se a venda é feita em mercados formais, o nível de exigência em termos informacionais aumenta. Abas (2016) constatou uma relação positiva entre os diversos canais de venda da produção, indicando que cada local de venda influenciava o nível de exigências ao produtor, isso em termos de qualidade e quantidade do produto.

A determinação das variáveis que maximizarão a probabilidade de os produtores ter acesso ou não é dada pela seguinte equação de logit.

$\operatorname{Logit}(P i)=\operatorname{Ln}\left(\frac{p i}{1-p i}\right)=\beta 0+\beta 1 \mathrm{NEP}+\beta 2 \mathrm{EPH}+\beta 3 \mathrm{SRF}+\beta 4 \mathrm{TAF}+\beta 5 \mathrm{TAP}+\beta 6 \mathrm{AT}+\beta 7 \mathrm{DLPM}+$

$+\beta 8 \mathrm{PDC}++\beta 9 \mathrm{RBP}+\beta 10 \mathrm{RP}+\beta 11 \mathrm{UPT}+\beta 12 \mathrm{PC} / \mathrm{A}+\beta 13 \mathrm{IP}+\beta 14 \mathrm{Sexo}+\beta 15 \mathrm{UTN}+\beta 16 \mathrm{TAF}+\beta 17 \mathrm{GP}+\beta 18 \mathrm{FUC}+\mu \mathrm{i}$

Em que: Pi é a probabilidade de ocorrência do evento; 1-Pié a probabilidade complementar

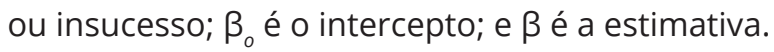

\subsection{Modelo Econométrico Logit Binário}

A regressão logística é uma regressão múltipla, com variável dependente categórica dicotômica e variáveis preditivas (explicativas ou independentes) contínuas ou categóricas. A resposta na regressão logística é expressa por meio de uma probabilidade de ocorrência (Gujarati \& Porter, 2011; Wooldridge, 2011).

Se a variável dependente for categórica, a equação será:

$y_{i}^{*}=\beta 0+X 1 \beta 1+X 2 \beta 2+X 3 \beta 3+X 4 \beta 4+X 5 \beta 5+X k \beta k+\mu$

Em que: $Y^{*}$ é a variável dependente binária; $i=1, \ldots ., \mathrm{n} ; \beta$ é o parâmetro; $X_{1}, X_{n}$ é conjunto de variáveis explicativas; e $\mu$ é o erro aleatório.

Os valores observados devem estar relacionados com a seguinte condição:

$\mathrm{Y}=0$, se $y_{i}^{*} \leq 0$

$\mathrm{Y}=1$, se $y_{i}^{*}>0$

Se o $\mu$ for simetricamente distribuído, resultará em: 


$$
\begin{aligned}
& \operatorname{Prob}(\mathrm{Yi}=1)=\operatorname{Prob}\left(y_{i}^{*}>0\right)=\operatorname{Prob}(\mu \mathrm{i}>-\mathrm{Xi} \beta) \\
& \operatorname{Prob}(\mathrm{Yi}=0)=\operatorname{Prob}\left(y_{i}^{*}=0\right)=\operatorname{Prob}(\mu \mathrm{i} \leq-\mathrm{Xi} \beta)
\end{aligned}
$$

A regressão logística é dada pela seguinte equação:

$\operatorname{logit}[p(x)]=\ln \left[\frac{p(x)}{1-p(x)}\right]=\beta 0+X 1 \beta 1+X 2 \beta 2+X 3 \beta 3+X 4 \beta 4+\beta k X k, i+\mu$

Em que:

$$
\begin{aligned}
& p(x)=\frac{\exp (\alpha+\beta x)}{1+\exp (\alpha+\beta x)} \\
& q=1-p(x)=1-\frac{\exp (\alpha+\beta x)}{1+\exp (\alpha+\beta x)}
\end{aligned}
$$

$p(x)$ é a probabilidade de ocorrência e $q$ é a probabilidade de insucesso.

O $p(\mathrm{x})$ deve ser linear nos parâmetros. O método para estimação dos estimadores betas, a partir do conjunto de dados na regressão logística, é a máxima verossimilhança. Esse método permite uma combinação de coeficientes que maximiza a probabilidade de a amostra ter sido observada (Agresti, 2007). A função da verossimilhança para um conjunto de dados $(x, y)$ é dada pela seguinte expressão:

$$
\pi(X i)^{Y i}[1-\pi(X i)]^{1-Y i}
$$

Se as observações assumidas forem independentes, logo a função de verossimilhança será obtida pela seguinte equação:

$$
\ell(\beta)=\prod \pi(X i)^{Y i}[1-\pi(X i)]^{1-Y i}
$$

Ou pela seguinte equação:

$$
L(\beta)=\prod \frac{\left[\mathrm{e}^{\alpha+\beta x}\right]^{\mathrm{yi}}}{\left[1+\mathrm{e}^{\alpha+\beta x}\right]} \times \frac{[1]^{1-y i}}{\left[1+\mathrm{e}^{\alpha+\beta x}\right]}
$$

Contudo, matematicamente é fácil trabalhar com o log da Equação 11. Assim, a expressão de máxima verossimilhança final logaritmizada será dada por:

$$
L(\beta)=\ln [\ell(\beta)]=\sum\{\mathrm{yi} \ln [\pi(\mathrm{xi})]+(1-\mathrm{yi}) \ln [1-\pi(\mathrm{xi})]\}
$$

Para o cálculo de valor de $\beta$ que maximiza $L(\beta)$, aplicam-se as derivadas do $L(\beta)$ em função do $\beta_{0}$ e $\beta_{1}$ e, em seguida, iguala-se o resultado a zero, da seguinte maneira:

$$
d L \frac{\beta}{d \beta j i}=\Sigma[\mathrm{yi}-\pi(\mathrm{xi})]=0
$$

$d L \frac{\beta}{d \beta j i}=\sum \mathrm{xi}[\mathrm{yi}-\pi(\mathrm{xi})]=0$

Para $\mathrm{j}=1,2$ e $\mathrm{k}=0,1,2 \ldots \ldots . . . \mathrm{p}$, com $\mathrm{x}_{01}=1$ para indivíduo. 
O valor $\beta$ obtido da derivação que é igualado a zero é chamado de estimador de máxima verossimilhança e é representado por beta chapéu. A interpretação dos resultados das regressões logit é feita por meio dos efeitos marginais (ou elasticidades). $O$ efeito marginal no modelo logit é representado pela equação (Gujarati \& Porter, 2011; Wooldridge, 2011):

$E M=\left[\frac{e^{\beta x}}{1+e^{\beta x}}\left(1-\frac{e^{\beta x}}{1+e^{\beta x}}\right)\right] \beta$ ou $E M=(p q) \beta$

Em que $x$ é ponto médio de cada variável; e $\beta$ é o coeficiente estimado.

\section{Resultados e discussões}

Constatou-se na pesquisa que $34,9 \%$ dos produtores tinham entre 19 e 40 anos, $27,6 \%$, entre 40 e 50 anos, 34,4\%, entre 50 e 70 anos, e, por fim, 3,1\%, entre 70 e 85 anos. A idade ativa em Moçambique, segundo o Ministério de Trabalho, Emprego e Segurança Social (MTESS) (Moçambique, 2016) e o Instituto Nacional de Estatística (2017b), ia de 15 a 64 anos. Com o avanço da idade, muitos produtores abandonavam a atividade agrícola, deixando os filhos como gestores. O Instituto Nacional de Estatística (2017a) aponta que a taxa de participação em idade laboral por área foi de 92,3\% para a rural e 7,7\% para a urbana.

Em relação ao sexo, do total dos pesquisados, 53,6\% eram do sexo feminino, e $46,4 \%$, do sexo masculino. O Instituto Nacional de Estatística (2017a) aponta que 52,2\% da população moçambicana era composta por indivíduos de sexo feminino. Para MTESS (Moçambique, 2017), o efetivo de mulheres na área rural envolvidas em atividades agrícolas foi de $84,9 \%$, enquanto o de homens, de $56 \%$.

O grau escolar na pesquisa foi de $49,7 \%$ dos produtores sem escolaridade, $24,7 \% \mathrm{com}$ primeiro grau completo, $16,9 \%$ com ensino médio, $6,5 \%$ com primeiro grau incompleto e, por fim, 1,3\% com curso superior completo. Para o Instituto Nacional de Estatística (2017a), a educação é um fator importante na sociedade, visto que, quanto maior for a escolaridade, maiores serão as possibilidades de evolução social. Verifica-se maior taxa de analfabetismo na área rural, sendo o grupo de mulheres o mais afetado.

Em relação ao tamanho da área produzida na pesquisa, constatou-se que $48,7 \%$ dos produtores tinham entre 0,1 e 1 ha, 27,6\%, entre 1 a 2 ha, 20,8\%, entre 2 a 10 ha, e 2,9\%, entre 10 a 20 ha. É possível notar que mais da metade dos produtores pesquisados (76,3\%) produzia em áreas compreendidas entre 0,1 a 2 ha. 0 tamanho da área encontrada está de acordo com a constatação feita por Freire (2001), de que o agricultor familiar cultiva em média uma área de 1,4 ha com uso de técnicas rudimentares e de baixo rendimento.

No que tange ao rendimento obtido na produção, 58,1\% produtores estavam satisfeitos, e $41,9 \%$, não satisfeitos. A não satisfação pelo rendimento obtido, considerado baixo pelos produtores, estava relacionada com a falta de boa gestão de água para irrigar as culturas. Outro fator da insatisfação foi a falta de informação, pois os produtores ficaram desprovidos de conhecimentos relacionados a certas tecnologias altamente produtivas.

Os produtores, por unanimidade (100\%), quando perguntados sobre infraestruturas existentes e os tipos das estradas, responderam que estas eram ruins e de terra batida, que se tornavam lamacentas nos dias chuvosos e impediam o trânsito de veículos para escoar a produção. A Organização das Nações Unidas para Alimentação e Agricultura (2013) e a USAID (United States Agency for International Development, 2015) caracterizam os estados das vias que ligam a região rural à urbana em Moçambique como precária e de difícil acesso, pois o 
mau estado das estradas provoca grandes perdas de produtos em trânsito das áreas rurais para a urbana.

Dos riscos associados à produção de hortaliças, a resposta foi consensual, uma vez que $100 \%$ dos produtores apontaram como riscos as condições climáticas, preços de insumos de produção, preço de venda, pragas e doenças. Para Buainain \& Silveira (2017), o risco da atividade agropecuária pode ser definido como uma medida de dispersão dos possíveis resultados que o agricultor pode obter em relação a um resultado esperado, por causa da interferência de fatores aleatórios ou imprevistos.

Todos os produtores envolvidos responderam que a comercialização era feita sem nenhum vínculo contratual e se baseava em relações interpessoais, confiança, na propriedade, vendas à beira da estrada, em domicílios e, às vezes, em feiras locais, mostrando que os produtores estavam inseridos em mercado de proximidade. Havia produtores que se manifestaram a favor da venda por contratos, enquanto a maioria preferia a venda sem vínculo contratual, porque se sentiam donos da produção e não precisavam enfrentar exigências padronizadas na qualidade e quantidade do produto. A atitude tomada pelos produtores vai de acordo com a constatação feita por Haber et al. (2015), de que a maior parte das transações de produtos agrícolas se baseia nas relações entre pessoas e se desenvolve de uma forma quase integralmente "informal". Outra descrença no uso de contratos é em razão da existência de oportunismo por parte das entidades com as quais o contrato é celebrado.

Apurou-se que $95,1 \%$ dos produtores concordavam que o sucesso na comercialização passava primeiro por ter acesso à informação sobre a produção e a comercialização, e 4,9\% eram indiferentes, isto é, não concordavam nem discordavam. Instituto Interamericano de Cooperacione para la Agricultura (2008) e Sato et al. (2006) afirmam que, quando a informação é acessível aos produtores, o processo de comercialização é transparente, ou seja, a formação do preço ao consumidor ocorre no fluxo do produtor ao consumidor, e a presença de intermediários interfere na distribuição das parcelas ou margens que cabem a cada agente participante dessa cadeia.

Dos pesquisados, 62,5\% tinham acesso à informação sobre a produção e a comercialização, como informação sobre as técnicas produtivas melhoradas na produção, sobre a concorrência sul-africana e sobre o lugar de venda da produção, preço dos insumos e preço de venda ao consumidor. Os demais (37,5\%) responderam que não tinham acesso à informação necessária como queriam e, quando conseguiam, era de forma superficial, isto é, dependiam de forma completa da intervenção do intermediário para comercializar a produção, assim como para Ihes dar notícias sobre os acontecimentos do mercado (de insumos e consumidor).

Na pesquisa, 54,9\% dos produtores tinham como fonte de referência de apoio à produção e comercialização de hortaliças a informação obtida pelos produtores vizinhos e intermediários, enquanto 45,1\% apenas usavam como informação de referência produtores cujas unidades de produção estivessem localizadas nas proximidades vizinhas. Os produtores optavam por essas fontes de informação como estratégia para garantir o escoamento da produção aos mercados de consumidores.

\subsection{Análises econométricas}

O modelo econométrico delineado na pesquisa acomodava 18 variáveis, sendo que algumas foram retiradas do modelo (Tabela 2) por causa de problemas relacionados com a multicolinearidade. Santana (2003), Gujarati \& Porter (2011), Wooldridge (2011) apontam como uma das formas utilizadas para a identificação da multicolinearidade a matriz de correlação 
simples entre as variáveis independentes da regressão. A presença da multicolinearidade faz com que as variâncias e os desvios-padrão tenham valores numéricos altos e grandes intervalos de confiança, levando os estimadores a uma não significância e a um alto valor de $\mathrm{R}^{2}$ (coeficiente de determinação), com poucas estatísticas significantes e alguns parâmetros com sinais trocados. Como meios correcionais desse problema, tem-se não fazer nada, retirar a variável colinear ou aumentar o tamanho da amostra.

Três variáveis na pesquisa foram retiradas do modelo por causa de problemas relacionados com a multicolinearidade, tais como: renda bruta, uso de pacote tecnológico e participação em dias de campo. A renda bruta apresentou um coeficiente de correlação considerado alto $(r=0,87)$ quando relacionado com o rendimento da produção. $O$ uso de pacote tecnológico apresentou uma relação moderada $(r=0,65)$ quando relacionado com a variável assistência técnica e obteve um coeficiente $r=0,76$ quando relacionado com a variável participação em dias de campo.

Em termos de aceitação dos resultados pelo modelo logitapresentado, infere-se o seguinte: 0 modelo em geral foi significativo a $p<0,05$, e 57,5\% da variável dependente (acesso à informação) é explicada ou influenciada pelas independentes em função da amostra colhida (Tabela 2). O acerto total do modelo foi de $83 \%$, sendo que esse valor corresponde ao percentual de acerto na classificação do acesso ou não acesso à informação pelos produtores de hortaliças. O teste de Hosmer-Lemeshow teve um valor de qui-quadrado igual a 5,9259, com um p-valor de 0,6555 , não significativo, fato que mostra a não existência de diferenças significativas entre os valores preditos e observados. Assim, o modelo é ideal e fornece boa explicação do que se observa na pesquisa.

As variáveis significativas foram a idade, se pertence a uma cooperativa ou associação de produtores, a experiência na produção, a satisfação com o retorno financeiro, a assistência técnica, o acesso ao crédito, o rendimento produzido por safra, o uso do telefone na negociação, a via usada para comercialização e a gestão da produção (Tabela 2).

A idade do produtor foi estatisticamente significativa a $5 \%$ de probabilidade $(p<0,05)$, com sinal negativo, indo de acordo com o esperado na pesquisa, isto é, a cada aumento em uma unidade na idade do produtor, havia uma diminuição em termos probabilísticos ao acessar a informação para fins comerciais nas hortaliças, ou, ainda, quando mais velho fosse o produtor, menos chance de ele procurar informação para comercializar a sua produção. $O$ efeito marginal da variável indica que, com o aumento em um ano na idade do produtor, ocorre uma diminuição da probabilidade em acessar a informação em 0,44\%.

Tabela 2. Resultados dos parâmetros estimados do modelo de regressão logística binária tendo como variável dependente o acesso à informação

\begin{tabular}{|c|c|c|c|c|c|}
\hline Variáveis & $\begin{array}{l}\text { Coeficiente } \\
\text { ( } \beta)\end{array}$ & $\begin{array}{l}\text { Erro } \\
\text { padrão }\end{array}$ & z-Estatístico & P-valor & $\begin{array}{c}\text { Efeito } \\
\text { marginal } \\
(\%)\end{array}$ \\
\hline C & $-9,491774$ & 2,312668 & $-4,104253$ & $0,0000^{*}$ & $-0,072$ \\
\hline Idade do produtor & $-0,044744$ & 0,021192 & $-2,111403$ & $0,0347^{* *}$ & $-0,44$ \\
\hline Sexo & 0,034363 & 0,394716 & 0,087058 & 0,9306 & 0,86 \\
\hline $\begin{array}{l}\text { Pertence à cooperativa/ } \\
\text { associação }\end{array}$ & 2,466467 & 0,409906 & 6,017149 & $0,0000 *$ & 42 \\
\hline Tamanho do agregado familiar & $-0,014807$ & 0,060343 & $-0,245384$ & 0,8062 & $-0,37$ \\
\hline Tamanho da área produzida & 0,099189 & 0,095337 & 1,040405 & 0,2982 & 2,45 \\
\hline $\begin{array}{l}\text { Experiência na prod. de } \\
\text { hortaliças }\end{array}$ & 0,043503 & 0,017309 & 2,513363 & $0,0120^{* *}$ & 0,86 \\
\hline
\end{tabular}

* significativo a $1 \%$ de probabilidade; ** significativo a $5 \%$ de probabilidade; *** significativo a $10 \%$ de probabilidade. 
Tabela 2. Continuação...

\begin{tabular}{|c|c|c|c|c|c|}
\hline Variáveis & $\begin{array}{c}\text { Coeficiente } \\
\text { ( } \beta)\end{array}$ & $\begin{array}{c}\text { Erro } \\
\text { padrão }\end{array}$ & z-Estatístico & P-valor & $\begin{array}{c}\text { Efeito } \\
\text { marginal } \\
(\%)\end{array}$ \\
\hline Distância do local da produção & 0,003081 & 0,004527 & 0,680665 & 0,4961 & 0,077 \\
\hline $\begin{array}{l}\text { Satisfação com o retorno } \\
\text { financeiro }\end{array}$ & 1,186411 & 0,385415 & 3,078268 & 0,0021 * & 27,3 \\
\hline Assistência técnica & 0,834667 & 0,445298 & 1,874402 & $0,0609^{* * *}$ & 19 \\
\hline Acesso ao crédito & 1,213139 & 0,401846 & 3,018916 & $0,0025^{*}$ & 28,3 \\
\hline Rendimento da produção & $-0,017764$ & 0,009765 & $-1,819220$ & $0,0689 * * *$ & $-0,44$ \\
\hline Uso de telefone na negociação & 3,334093 & 0,436306 & 7,641644 & $0,0000 *$ & 37 \\
\hline Forma usada na comercialização & 0,337617 & 0,200151 & 1,686808 & $0,0916 * * *$ & 6,7 \\
\hline Gestão da propriedade & 1,201231 & 0,379946 & 3,161584 & $0,0016^{*}$ & 0,36 \\
\hline Nível escolar do produtor & $-0,045978$ & 0,162728 & $-0,282548$ & 0,7775 & $-1,14$ \\
\hline McFadden R-squared & 0,574800 & \multicolumn{2}{|c|}{ Prob (LR statistic) } & 0,000000 & \\
\hline Obs with $\mathrm{Dep}=0$ & 144 & \multicolumn{2}{|c|}{ Total obs } & 384 & \\
\hline Obs with Dep=1 & 240 & & & & \\
\hline
\end{tabular}

* significativo a $1 \%$ de probabilidade; ** significativo a $5 \%$ de probabilidade; *** significativo a $10 \%$ de probabilidade.

O resultado encontrado na pesquisa é corroborado pelo estudo feito por Santos Filho (2008), no qual constatou que a idade era um fator preponderante na determinação de pobreza rural e urbana em Santa Catarina, isto é, a pobreza era mais acentuada em indivíduos idosos quando comparados com os mais jovens. Carrer et al. (2013) chegaram ao mesmo resultado, constatando uma relação negativa entre a idade dos pecuaristas e o acesso ao crédito.

A variável pertencente à cooperativa/associação de produtores obteve uma estatística significativa a $1 \%$ de probabilidade $(p<0,01)$ e positiva. Isso demonstra que, quanto mais organizados (em cooperativas e associações) estivessem os produtores em prol de um objetivo em comum, maiores as chances de obterem o acesso à informação para comercializar a produção. O efeito marginal da variável indica que produtores organizados em associações ou cooperativas tinham um aumento na probabilidade de terem acesso à informação agrícola sobre a produção e a comercialização em $42 \%$.

O resultado está de acordo com a pesquisa feita por Theophilus et al. (2019), em um estudo feito em Gana, cujo título era Determinants of the extent of adoption of maize production technologies in Northern Ghana. Já Aikin (2014) e Rajendran et al. (2016) constataram que, quanto mais organizados estivessem os produtores em cooperativas/associações, mais chances teriam em adotar tecnologias produtivas na produção de culturas, culminando com o aumento da produção e produtividade.

A variável experiência na produção das hortaliças foi estatisticamente significativa a $5 \%$ de probabilidade $(p<0,05)$ e positiva. O acesso à informação na comercialização das hortaliças está relacionado com a experiência do produtor na produção, isto é, quanto mais experiente fosse o produtor, maior seria a chance de obter informação. O efeito marginal da variável indica que, aumentando em um ano no tempo de produção, a probabilidade de se ter acesso à informação na comercialização das hortaliças aumenta em $0,86 \%$. Resultado semelhante foi encontrado por Nchembi (2017) e Dhraiefa et al. (2018), ao constatarem que um maior tempo de produção aumentava a probabilidade dos produtores em aderir à tecnologia altamente produtiva agrícola.

A variável satisfação com o retorno financeiro foi estatisticamente significativa a $1 \%$ de probabilidade $(p<0,01)$ e positiva, mostrando que, quanto mais satisfeito estivesse o produtor pelo retorno financeiro decorrente da atividade comercial de hortaliças, maior a probabilidade 
de ele ter acesso à informação sobre a comercialização de hortaliças. O efeito marginal de variável indica que, quanto mais satisfeito estivesse o produtor pelo retorno financeiro na comercialização de hortaliças, a probabilidade em acessar a informação para aprimorar a atividade e buscar mais eficiência gerando lucros aumentaria em 27,3\%.

Em relação ao resultado, Padilha Junior (2006) afirma que não adianta haver uma eficiência produtiva dentro da propriedade rural, com alta produtividade e custos baixos, se, ao se colocar a produção dentro de um sistema de comercialização, perde-se toda a vantagem comparativa e competitiva. Um encaminhamento organizado desde a produção até a comercialização agropecuária permite que os consumidores finais obtenham os produtos com as características desejadas, agregando valor à produção e à maximização de lucros.

A variável assistência técnica foi estatisticamente significativa a 10\% de probabilidade $(p<0,1)$ e com sinal positivo, indicando, assim, uma contribuição positiva para o acesso à informação na comercialização agrícola, isto é, a assistência técnica munia os produtores de ferramentas necessárias, desde o acesso à informação até o acesso a novas tecnologias produtivas. $O$ efeito marginal da variável indica que, aumentando a frequência das visitas de assistência técnica, a probabilidade de se ter acesso à informação na comercialização de hortaliças aumentaria em 19\%. Resultados que corroboram esta pesquisa foram encontrados por Ghimire et al. (2015), Melesse (2018), Theophilus et al. (2019) e Lanna et al. (2011), ao concluírem que as visitas técnicas desempenhavam um papel importante na adoção de novas tecnologias e no aumento do rendimento da produção.

A variável acesso ao crédito foi estatisticamente significativa a $1 \%$ de probabilidade $(p<0,01)$ e com sinal positivo, indicando que, quanto mais crédito tivessem os produtores, mais chances tinham de acessar a informação para materializar o processo de comercialização. $O$ acesso ao crédito constitui uma força motriz na produção de hortaliças em Moçambique, assim como em outros países. O efeito marginal indica que a maior disponibilidade de crédito aos produtores aumentaria a probabilidade destes em acessar informações sobre a comercialização das hortaliças em 28,3\%. Resultado semelhante foi encontrado por Oliveira et al. (2005) e Theophilus, Melesse (2018), ao constatarem que o acesso ao crédito era o fator determinante para o uso de tecnologias altamente produtivas.

A variável rendimento de produção obtido por safra foi estatisticamente significativa, com 10\% de probabilidade $(p<0,1)$ e sinal negativo, apontando que, quanto maior fosse o rendimento, menor seria o acesso à informação na produção e comercialização das hortaliças. $O$ efeito marginal da variável indica que, com o aumento de uma tonelada no rendimento produzido, ocorreria uma diminuição no acesso da informação na comercialização de hortaliças em 0,44\%. Resultado semelhante, que corrobora esta pesquisa, foi encontrado por Nchembi (2017), ao constatar que o aumento do rendimento produzido agia de forma contrária à adoção de variedades melhoradas.

A variável uso de telefone nas negociações foi estatisticamente significativa a $1 \%$ de probabilidade $(p<0,01)$ e positiva, indicando assim que, com o uso frequente de telefone pelos produtores para negociar a produção, haveria mais chances de acessar a informação. $O$ uso do telefone para negociar a produção agrega valor no tempo e no espaço, gerando eficiência produtiva. O efeito marginal da variável indica que, com o aumento da frequência no uso de telefone na negociação da produção, a probabilidade de se ter acesso à informação sobre a comercialização aumentaria em $37 \%$. A fim de corroborar os resultados desta pesquisa, Dias (1997) e Aikin (2014) obtiveram o mesmo resultado, ao constatarem que produtores que usavam o telefone para mediar o processo comercial tinham mais chances de obter informações sobre a comercialização quando comparados com os que não faziam. 
A variável forma usada para comercialização da produção foi estatisticamente significativa a $10 \%$ de probabilidade $(p<0,1)$ e positiva, apontando que, quanto mais formal e estruturado fosse o local de venda da produção, haveria maiores possibilidades de se ter o acesso à informação para a comercialização das hortaliças. Em Moçambique, os produtores negociavam a produção em mercados informais, em feiras de agronegócios e em mercados formais. O efeito marginal da variável indica que, quanto mais estruturado e formal fosse o canal pelo qual a comercialização seria realizada, a probabilidade em ter acesso à informação para garantir o escoamento da produção aumentaria em 6,7\%. De acordo com Padilha Junior (2006), Barros (2007) e Callado (2011), a quantidade de informação exigida para garantir que a comercialização aconteça tem a ver com o local onde a transação irá ocorrer.

A variável gestão da propriedade foi estatisticamente significativa a $1 \%$ de probabilidade $(p<$ 0,01) e com sinal positivo, evidenciando que, na presença de uma boa gestão, haveria maiores possibilidades de se ter acesso à informação para tornar efetiva a atividade de produção e comercialização das hortaliças. O efeito marginal da variável indica que, quanto mais eficiente fosse o sistema de gestão da propriedade, a probabilidade do acesso à informação ao produtor aumentaria em 0,36\%. Esse resultado vai de acordo com a constatação feita por Callado (2011) e Abas (2016) de que uma boa gestão em propriedades rurais facilita a escolha de melhores alternativas e proporciona um nível de autossuficiência aos produtores.

\section{Conclusão}

O objetivo central da pesquisa foi identificar e analisar os fatores determinantes para o acesso à informação por produtores de hortaliças na região sul de Moçambique. O perfil dos produtores envolvidos na produção da pesquisa foi de pessoas com idade compreendida entre 19 e 85 anos, dos quais 50\% não tinham grau de formação escolar, 46\% eram homens e 54\% eram mulheres, que cultivavam em pequenas áreas de forma intensiva.

O produtor moçambicano se depara com dificuldades no acesso aos mercados para a comercialização da sua produção, fato que pode estar relacionado às suas características socioeconômicas específicas e ainda à conjuntura econômica do país em geral. As dificuldades socioeconômicas, que têm como principal característica o elevado índice de pobreza, fazem da comercialização agrícola uma atividade que ocorre em regime informal. Assim sendo, torna-se imprescindível a adoção de estratégias relacionadas à promoção de iniciativas para valorização de produtos e de acesso aos mercados, as quais sejam ligadas à forma de organização social e, em simultâneo, criem mecanismos de construção social de mercados diversificados e de qualificação dos produtos.

O acesso à informação ao produtor rural na produção e comercialização das hortaliças é feito de forma assimétrica, pois nem todos possuem informação; assim, uma parte ainda depende do intermediário, fato que tem prejudicado o progresso e a sustentabilidade da atividade de forma social e econômica. A informação aos produtores de hortaliças é crucial e relevante, já que permite que eles se mantenham conectados com os acontecimentos de mercados de insumos e de consumo. Por outro lado, ao produtor familiar a informação é vital por ser fonte para a construção do conhecimento sobre técnicas produtivas e facilitar a comunicação entre os diversos agentes do mercado, contribuindo para a busca de eficiência e geração de renda.

A ausência da informação ao produtor de hortaliças tem gerado assimetria de informação e emergência de atitudes oportunistas por parte dos compradores, colocando o produtor em um ambiente de incertezas e racionalidade limitada. Outro aspecto não menos importante ligado ao processo produtivo e comercial de produtos agrícolas em Moçambique é o mau 
funcionamento das cadeias de valores, as quais, além de agregarem valores, melhorarem as perspectivas comerciais dos produtores e ainda aumentarem as margens de lucro, atuam em sentido contrário de não viabilidade da atividade por causa da falta de conectividade e coordenação entre os agentes nela envolvidos.

A comercialização das hortaliças em Moçambique é feita em um ambiente informal, em que grande parte das transações se baseia em relações interpessoais, criando espaço para o desenvolvimento de laços e afinidades entre os produtores e os consumidores. O mercado específico é o de proximidade, pois a forma usada pelo produtor para escoar a produção é por meio de entregas diretas, visitas aos domicílios e participação em feiras locais de agronegócios. Além disso, os produtores têm recebido em sua propriedade consumidores e intermediários.

Os resultados do modelo de regressão logit binário indicam as seguintes variáveis como significativas: idade do produtor; se pertence a uma cooperativa/associação de produtores; experiência na produção; satisfação com o retorno financeiro; assistência técnica; acesso ao crédito; rendimento da produção; uso de telefone na negociação; forma usada para comercializar a produção; e gestão da propriedade. As variáveis foram significativas e positivas, indicando que, com um aumento adicional em cada uma delas, haverá um aumento de probabilidade de os produtores terem acesso à informação na produção e comercialização das hortaliças. As variáveis idade e rendimento da produção foram significativas e negativas, evidenciando que, com um incremento adicional nelas, menor é a chance de se obter a informação.

A significância das variáveis como fatores determinantes ao acesso à informação na produção e comercialização das hortaliças deixa um desafio ao governo de Moçambique em elaborar políticas voltadas ao desenvolvimento, melhoria e criação de condições, de forma que elas estejam presentes na produção das hortaliças e acessíveis a ela e, em simultâneo, melhorem de forma substancial a vida social e o bem-estar dos produtores. Os produtores com características que se apresentam em forma de variáveis estatisticamente significativas no modelo têm vantagem no acesso à informação sobre a produção e a comercialização de hortaliças do que aqueles que não têm esse conjunto de características.

\section{Referências}

Abas, M. S. (2016). Factors influencing self-reliance in rice production, the case of small farmers in Bataan, Philippines. International Journal of Agricultural Technology, 12(1), 41-53.

Adio, E. O., Abu, Y., Yusuf, S. K., \& Nansoh, S. (2016). Use of agricultural information sources and services by farmers for improve productivity in Kwara State. Lincoln: University of Nebraska. Recuperado em 25 de maio de 2020, de https://digitalcommons.unl.edu/libphilprac/1456/

Agresti, A. (2007). An introduction to categorical data analysis (2nd ed.). Gainesville: Department of Statistics, University of Florida.

Aikin, I. (2014). The effect of access to and use of agricultural information on the livelihood of cocoa farmers (Thesis). Department of Agricultural Extension, School of Agriculture, College of Basic and Applied Sciences, University of Ghana, Ghana.

Alemu, D., Groote, H., \& Bacha, D. (2006). The role of market information system in improving rural livelihood and the status of the service in Ethiopia. Journal of Agricultural Economics, 6(1), 87-108.

As-Sunny, F., Huang, Z., \& Karimanzira, T. T. P. (2018). Investigating key factors influencing farming decisions based on soil testing and fertilizer recommendation facilities (STFRF), a case Study on Rural Bangladesh. Sustainability, 10(11), 1-24. 
Barreto, A. A. (1996). A eficiência técnica e econômica e a viabilidade de produtos e serviços de informação. Ciência da Informação, Brasilia, 25(3), 1-18.

Barros, G. S. A. C. (2007). Economia da comercialização agrícola. Piracicaba: Departamento de Economia, Administração e Sociologia, Escola Superior de Agricultura "Luiz de Queiroz", Centros de Estudos Avançados em Economia Aplicada, Universidade de São Paulo.

Batalha, M. O. (Coord.). (2014). Gestão agroindustrial: GEPAl - Grupo de estudos e pesquisas agroindustriais (3. ed.). São Paulo: Atlas.

Bati, B. (2017). Determinants of adoption of improved potato varieties by smallholder farmers in shashemene district, west arsi zone, oromia national regional state, Ethiopia(Msc Thesis). Haramaya University, Haramaya.

Buainain, A. M., \& Silveira, R. L. (2017). Manual de avaliação de riscos na agropecuária: um guia metodológico (1. ed.). Rio do Janeiro.

Callado, A. A. C. (Ed.). (2011). Agronegócio (3. ed.). São Paulo: Atlas.

Carrer, M. J., Souza Filho, H. M., \& Vinholis, M. M. B. (2013). Determinantes de demanda de crédito rural por pecuaristas de corte no estado de São Paulo. Revista de Economia e Sociologia Rural, 51(3), 455-478.

Couto, A. C. L., \& Alves, A. F. (2013). Fatores socioeconômicos associados à pobreza: uma análise para a área rural do Brasil (2004-2009). In VIII SOBER.

Dhraiefa, M. Z., Bedhiaf-romdhania, S., Dhehibib, B., Oueslati-zlaouia, M., Jebalia, O., \& Ben Youssefa, S. (2018). Factors affecting innovative technologies adoption by livestock holders in arid area of Tunisia. New Medit, 18(4).

Dias, R. L. (1997). O papel de informação de mercado na comercialização de hortigranjeiros no Distrito Federal (Dissertação de mestrado). Universidade Federal de Viçosa, Viçosa.

Freire, M. (2001). Apontamentos de agricultura geral(124 p.). Maputo: Faculdade de Agronomia e de Engenharia Florestal, Universidade Eduardo Mondlane.

Ghimire, R., Huang, W., \& Shrestha, R. B. (2015). Factors affecting adoption of improved rice varieties among rural farm households in central Nepal. Rice Science, 22(1), 1.

$<$ bok>Gujarati, D. N., \& Porter, D. C. (2011). Econometria básica (5. ed. Porto Alegre: AMGH.</bok>

Haber, L. L., Ecole, C. C., Bowen, W., \& Resende, F. V. (2015). Horticultura em Moçambique Características, Tecnologias de Produção e de Pós-Colheita. Brasília, DF: Embrapa. Recuperado em 25 de maio, de https://www.infoteca.cnptia.embrapa.br/infoteca/handle/doc/1029218.

Instituto Interamericano de Cooperação para a Agricultura - IICA. (2016). Sistemas de informação de mercados agrícolas SIMA do IICA. São José, Costa Rica: IICA. Recuperado em 25 de maio de 2020, de repositorio.iica.int/bitstream/11324/6448/4/BVE17079124p.pdf

Instituto Interamericano de Cooperacione para la Agricultura - IICA. (2008). Seguro agropecuário. Poderosa herramienta para gobiernos y agricultores. Ano 4/ II Etapa. Recuperado em 25 de maio de 2020, de http://repiica.iica.int/docs/B0730e/B0730e.PDF

Instituto Nacional de Estatística - INE. (2017a). Mulheres e homens em Moçambique. Moçambique. Recuperado em 25 de maio de 2020, de www.ine.gov.mz

Instituto Nacional de Estatística - INE. (2017b). Resultados definitivos do censo 2017. Recuperado em 25 de maio de 2020, de http://www.ine.gov.mz/iv-rgph-2017/mocambique/apresentacaoresultados-do-censo-2017-1

Israel, G. D. (1992). Sampling the evidence of extension program impact. Program Evaluation and Organizational Development, IFAS, University of Florida. Recuperado em 25 de maio 
de 2020, de https://pdfs.semanticscholar.org/0917/234e6cef056d2df0ed155f9ee60c9b6 57efd.pdf

Lachica, R., Karabeg, D., \& Rudan, S. (2008). Quality, relevance and importance in information retrieval with fuzzy semantic networks. Norway. Recuperado em 25 de maio de 2020, de https://folk.uio.no/dino/KF/Lachica-Karabeg08.pdf

Lanna, G. B. M., Teixeira, E. C., \& Reis, R. P. (2011). Determinantes da adoção da tecnologia de despolpamento na Cafeicultura: estudo de uma região produtora da zona da mata de Minas Gerais. Organizações Rurais \& Agroindustriais, 3(3), 352-362.

Lemessa, S. D., Daksa, M. D., Alemayehu, M., \& Dechassa, N. (2019). Adoption Spells of Improved Potato Varieties by Smallholder Farmers in Eastern Ethiopia: The Duration Approach. Problems of World Agriculture. Scientific Journal Warsaw University of Life Sciences, 19(2), 103-118.

Machado, M. (2014). Banco oportunidade de Moçambique. Serviço de consultoria para conduzir um estudo de mercado sobre volumes, preços, novos produtos e produção sob contrato na cadeia de valor hortícola no Centro de Moçambique (Relatório final). Recuperado em 25 de maio de 2020, de https://docplayer.com.br/4622327-Maputo-20-2014-marco-machado.html

Melesse, B. A. (2018). Review on factors affecting adoption of agricultural new technologies in Ethiopia. Journal of Agricultural Science and Food Research, 9(3), 9-3.

Ministério de Agricultura - MINAG. (2011). Programa de Investimento do Sector Agrário (PROAGRI II) - Avaliação Ambiental Estratégica, Maputo. Recuperado em 25 de maio, de http://www. minag.gov.mz/images/stories/pdf_files/aae.pdf.

Moçambique. Ministério de Agricultura e Segurança Alimentar - MASA. (2017). Programa Nacional de Mecanização Agrário. Maputo.

Moçambique. Ministério de Trabalho, Emprego e Segurança Social - MTESS. (2016). Boletim informativo de mercado de trabalho 2015/2016. Recuperado em 25 de maio de 2020, de http://www.mitess.gov.mz/boletins-estatisticos

Nchembi, N. S. (2017). Factors influencing the use of improved maize seed technology in Kilosa district in Tanzania (MSc. thesis). Lilongwe University of Agriculture and Natural Resources, Malawi.

O'Donnell, S. W. (2009). The importance of information customer relationship management is key. Enterprise Resource Planning, 59(5).

Oliveira, M. A. S., Khan, A. S., \& Lima, P. V. P. S. (2005). Adoção tecnológica e seus condicionantes: o caso da bananicultura no agro polo Cariri-CE. Revista de Economia e Agronegócio, 3(3).

Organização das Nações Unidas para Alimentação e Agricultura - FAO. (2013). Primeiro relatório sobre a agricultura urbana e periurbana (Cidades mais verdes na África). Roma. Recuperado em 25 de maio de 2020, de http://www.fao.org/3/i3002p/i3002p.pdf

Padilha Junior, J. B. (2006). Comercialização de produtos agrícolas. Curitiba.

Paulo, A. M. (2011). Determinantes de renda das famílias rurais em Moçambique entre 2005 a 2008 (Dissertação de mestrado). Universidade Federal de Viçosa.

Paustian, M., \& Theuvsen, L. (2016). Adoption of precision agriculture technologies by German crop farmers, Springer Science Business Media New York. Precision Agriculture, 18, 701-716.

Rajendran, N., Tey, Y. S., Brindal, M., Ahmad Sidique, S. F., Shamsudin, M. N., Radam, A., \& Abdul Hadi, A. H. I. (2016). Factors influencing the adoption of bundled sustainable agricultural practices: a systematic literature review. International Food Research Journal, 3(5), 2271-2279. 
Rocha Junior, W. F. (2004). A nova economia institucional revisitada. Revista de Economia e Administração, 3(4), 301-319.

Rocha Junior, W. F., \& Ribeiro, M. C. P. (2011). Institutional environment and contracts: a case study in the agroindustrial system of broiler factory farming in the west of the state of Paraná, Brazil. Recuperado em 25 de maio de 2020, de http://www.sideisle. it/ocs2/index. php/SIDE/side-isle2011/paper/view/718

Santana, A. C. (2003). Métodos quantitativos em economia: elementos e aplicações (484 p.). Belém: Universidade Federal Rural da Amazônia.

Santos Filho, J. I. (2008). Fatores determinantes da pobreza rural e urbana em Santa Catarina. In I/ Encontro de Economia Catarinense: Demografia e Mercado de Trabalho. Chapecó, SC.

Sato, G. S., Martins, S. S., De Carvalho, Y. M. C., Milani, A. A., \& Cunha, R. P. (2006, julho 23-27). Fluxo De Comercialização De Hortaliças Produzidas Na Região Alto Cabeceiras Do Tietê. In 44th Congress. Fortaleza, Ceará: Sociedade Brasileira de Economia, Administracao e Sociologia Rural (SOBER). Recuperado em 25 de maio, de https://ideas.repec.org/p/ags/ sobr06/148149.html.

Schneider, I. A. (1990). Produção e comercialização agrícola, fontes de informação e relevância. Revista de Economia e Sociologia Rural, 28(3), 69-86.

Schneider, S. (2016). Mercados e agricultura familiar. In F. C. Marques, M. A. Conterato \& S. Schneider (Eds.), Construção de mercados e agricultura familiar: desafios para o desenvolvimento rural (pp. 93-140). Porto Alegre.

Silva, J. G. (Ed.). (2019). Do Fome Zero ao Zero Hunger: uma perspectiva global. Roma: FAO.

Stiglitz, J. E. (2001). Information and the change in the paradigm in economics. New York: Columbia Business School, Columbia University.

Theophilus, K. A., Robert, A., \& Paul, S. M. (2019). Determinants of the extent of adoption of maize production technologies in Northern Ghana. African Journal of Agricultural Research, 14(19), 819-827.

United States Agency for International Development - USAID SPEED (2015). Janeiro de variações de preços $m$ Moçambique. Projeto de Relatório. Recuperado em 25 de maio, de www. speed-program.com/.../2015/.../2015-SPEED-Report-010-Cross-Boarder-Price.

Williamson, O. E. (1985). The economic institutions of capitalism. New York: Free Press.

Wooldridge, J. A. (2011). Introdução a econometria: uma abordagem moderna (4. ed.). São Paulo: Cengage Learning.

World Bank. (2018). Poverty and shared prosperity: piecing together the poverty puzzle. Recuperado em 25 de maio de 2020, de https://openknowledge.worldbank.org/bitstream/ handle/10986/30418/9781464813306.pdf 